Pacific Journal of Mathematics

A NOTE ON TOPOLOGICAL TRANSFORMATION GROUPS 


\title{
A NOTE ON TOPOLOGICAL TRANSFORMATION GROUPS WITH A FIXED END POINT
}

\author{
William J. Gray
}

Let $(X, T, \Pi)$ be a topological transformation group, where $X$ is a nontrivial Hausdorff continuum, and $T$ is a topological group which leaves an endpoint $e$ of $X$ fixed. Wallace showed that if $X$ is locally connected and $T$ is cyclic, $T$ has another fixed point. In a later paper, Wallace asked the following question: if $X$ is a peano continuum and $T$ is compact or abelian, does $T$ have another fixed point?

In 1952, Wang showed that if $X$ is arcwise connected and $T$ is compact, $T$ has another fixed point; Chu has recently extended this result by showing $T$ has infinitely many fixed points. Gray has shown that in the abelian case, the answer to Wallace's question is "no" (in general). However, if $T$ is a generative group, and if $X$ is arcwise connected, $T$ has another fixed point. In this paper we will generalize the last result. In fact, we show that if $X$ is arcwise connected or locally connected, and $T$ is a group of the form $A H$, where $H$ is a connected subgroup, and $A$ is an abelian group generated by a compact subset, and $A$ lies in the center of $T$, then $T$ has another fixed point. We will generalize several known theorems by studying ordered spaces similar to those introduced by Wallace in 1945; in particular, we will obtain a generalized solution of the compact group problem (Theorem 2).

2. In this section, $X$ will denote a compact Hausdorff space consisting of more than two points on which a reflexive, transitive, and antisymmetric order $\leqq$ is defined; if $z \in X$, let

$$
L(z)=\{x ; z<x\}, M(z)=\{x ; x \leqq z\}, N(z)=\{x ; z \leqq x\} .
$$

We assume that $\leqq$ satisfies the following conditions:

(a) The set $M(z)$ is closed.

(b) The set $L(z)$ is open, and $N(z)$ is closed.

(e) $X$ has a least element $e$ under $\leqq$.

(d) Each set $M(z)$ is a chain, i.e. $M(z)$ is simply ordered by $\leqq$.

(e) $X$ is directed by $\leqq$ in the following sense: if $x, y \in X$ and $x \neq e, y \neq e$, then there exists $z \neq e$ such that $z \leqq x$ and $z \leqq y$.

Wallace, [6], has proved:

(f) Each nonvoid closed subset of $X$ contains a maximal element under $\leqq$.

We show:

(g) If $C$ is a closed nonvoid subset of $X$ with $e \notin C$, we have 
$z \in X, z \neq e$, for which $z \leqq c$ for every $c \in C$.

Proof. If for some $z \in C, z \leqq c$ for every $c \in C$, we are finished. Otherwise, if $x, y \in C$ and $x \neq y$, choose $z_{x y} \neq e$ satisfying (e): $z_{x y} \leqq x$ and $z_{x y} \leqq y$. We show that the collection $\left\{L\left(z_{x y}\right) ; x, y \in C, x \neq y\right\}$ is an open cover of $C$. If $x \in C$, we have $y \in C$ for which $x \notin y$; it follows that $z_{x y}<x$, and hence $x \in L\left(z_{x y}\right)$. Since $X$ is compact and $C$ is closed in $X$, there is a finite subset $\left\{z_{i}, \cdots, z_{n}\right\}$ of the set $\left\{z_{x y} ; x, y \in C\right.$, $x \neq y\}$ for which $C \subset \cup\left\{L\left(z_{j}\right), 1 \leqq j \leqq n\right\}$; since $z_{j} \neq e$ for every $j$, by (e) we have $z \in X$ for which $z \neq e$ and $z \leqq z_{j}$ for $j=1, \cdots, n$. $z$ is the desired element of $X$.

By an order isomorphism: $X \rightarrow X$, we mean a homeomorphism which preserves $\leqq$. If $(X, T, \Pi)$ is a transformation group, we will assume that for each $t \in T$ the $t$-transition of $(X, T, \Pi)$ is an order isomorphism.

If $A \subset X$ and $B \subset X$, we write $A \leqq B[A<B]$ if, given $a \in A$ and $b \in B$, we have $a \leqq b[a<b]$.

LEMMA 2.1. Let $(X, T, \Pi)$ be a topological transformation group. If there is a closed nonempty $T$-invariant subset $A \subset X$ such that $e \notin A$, then $T$ has a fixed point other than $e$.

Proof. Let $A \neq \varnothing$ be any closed subset of $X$ such that $e \notin A$. Define

$$
M(A)=\cap\{M(\alpha) ; \alpha \in A\} .
$$

$M(A)$ is a closed chain, and $M(A)=\{z ; z \leqq A\}$. By $(\mathrm{g}), M(A)$ does not consist of $e$ alone. By (f), $M(A)$ contains a maximal element $\mathscr{M}(A)$. Since $M(A)$ is a chain, $\mathscr{C}(A)$ is the largest element of $M(A)$. If $t: X \rightarrow X$ is an order isomorphism, then $t \mathscr{C}(A)=\mathscr{C}(t A)$. Then if $A$ is $T$-invariant, $\mathscr{C}(A)$ is fixed under $T$. It is clear that $\mathscr{C}(A) \neq e$, so that the proof is complete.

Lemma 2.2. Let $(X, T, \Pi)$ be a transformation group. If there is a T-invariant chain $B \subset X$ which is not empty and does not consist of $e$ alone, then $T$ has a fixed point other then $e$.

Proof. The collection of closed sets $\{N(b) ; b \in B\}$ has the finite intersection property since $B$ is a chain. Hence the intersection, $N(B)$, of the $N(b)$ is not empty and is T-invariant since $B$ is. Because $e \notin N(B), N(B)$ satisfies the hypothesis of Lemma 2.1, and the proof is complete. 
LEMMA 2.3. Let $t_{1}, \cdots, t_{n}$ be commuting order isomorphisms: $X \rightarrow X$. Then the $t_{i}$ have a fixed point other than $e$ in common.

Proof. Let $z_{0}$ be a maximal element of $X$. If $A=\left\{z_{0}, t_{i}^{-1} z_{0} ; i=\right.$ $1, \cdots, n\}$, then $e \notin A$. By (e) we have $z_{1} \neq e, z_{1} \leqq A$. For each $i$, $\left\{z_{1}, t_{i} z_{1}\right\} \subset M\left(z_{0}\right)$. We let $T_{i}$ be the cyclic group generated by $t_{i}$ and $T=T_{1} T_{2} \cdots T_{n}$. Then for each $i, T_{i} z_{1}$ is a chain.

(1) If $s \in T$ and $t \in T$ such that $s z_{1}$ and $t z_{1}$ both compare to $z_{1}$, then $s z_{1}$ and $t z_{1}$ compare.

For if $s z_{1} \leqq z_{1}$ and $t z_{1} \leqq z_{1}$, the result follows from (d). If $s z_{1} \geqq z_{1}$ and $t z_{1} \geqq z_{1}$, apply the last case to $s^{-1} z_{1}$ and $t^{-1} z_{1}$ and use the fact that $T$ is abelian. The final case follows by transitivity of $\leqq$.

(2) Each element of $T z_{1}$ compares to $z_{1}$.

Let $t_{1}^{K_{1}} \cdots t_{n}^{K_{n}} z_{1} \in T z_{1}$, where the $K_{i}$ are integers. Then $t_{1}^{K_{1}} z_{1}$ compares to $z_{1}$. We proceed by induction. If $t_{1}^{K_{1}} \cdots t_{j}^{K} z_{1}$ compares to $z_{1}$, where $1 \leqq j \leqq n-1$, then since $t_{j+1}^{K j+1} z_{1}$ compares to $z_{1}$ also, $t_{1}^{K_{1}} \cdots t_{j+1}^{K_{j+1}} z_{1}$ compares to $z_{1}$; the desired result follows.

From (1) and (2) it follows that $T z_{1}$ is a chain. Now $e \notin T z_{1}$ so that Lemma 2.2 applies. The proof is complete.

A group $T$ is generative if $T$ is abelian and is generated by a compact neighborhood of the identity of $T$.

Theorem 1. Let $(X, T, \Pi)$ be a transformation group, where $T$ acts as a generative group of order isomorphisms on $X$. Then $T$ has a fixed point other than $e$.

Proof. Since $T$ is generative, it is known that $T$ has the form $K Z^{m} R^{n}$ where $Z$ and $R$ denote the integers and reals, respectively, with the usual topology, and $m$ and $n$ are nonnegative integers. Thus $T$ may be written in the form $C A$, where $C$ is compact and $A$ is a finitely generated abelian group. If $x$ is a fixed point of $X$ under $A$, with $x \neq e$, then $T x=C x$ is closed, $T$-invariant, and does not contain $e$. Hence Lemma 2.1 applies, and the proof is complete.

Note. Actually, in Theorem 1, we need only assume that the group $T$ is abelian and is generated by a compact set. For if then $C$ is a compact symmetric set which contains the identity of $T$ and generates $T$, let $x$ be a maximal element of $X$ and let $z \leqq C^{-1} x$, where $e \neq z$. Then $C z \subset M(x)$, hence $C z$ is a chain. Since $T$ is abelian, we may argue as in the proof of Lemma 2.3 and show that $C^{n} z$ is a chain for each positive integer $n$. Thus the set $\cup\left\{C^{n} z ; n=1,2, \cdots\right\}$ is a $T$-invariant chain not consisting of $e$ alone, and $T$ has a fixed point other than $e$. This proves 
Theorem 1'. If $(X, T, \Pi)$ is a transformation group, where $T$ is abelian and is generated by a compact subset, and if $T$ acts as a group of order isomorphisms on $X$, then $T$ has a fixed point other than $e$.

We now consider a strengthened form of axiom (e):

$\left(\mathrm{e}_{\mathrm{s}}\right) \quad X$ is strongly directed by $\leqq$ in the following sense: if $x, y \in X$ and $x \neq e, y \neq e$, then there is a $z \in X$ with $z \neq e$ for which $z<\{x, y\}$.

If $X$ is a space which satisfies (a)-(e) but does not satisfy $\left(e_{s}\right)$, then it is easy to see that there is an $x \in X$ with $x \neq e$ such that $t x=x$ for every order isomorphism $t: X \rightarrow X$. If $X$ satisfies $\left(\mathrm{e}_{\mathrm{s}}\right)$, then we have

$\left(\mathrm{g}_{\mathrm{s}}\right)$ If $C$ is a closed nonempty subset of $X$ with $e \notin C$, there is a $z \in X$ with $z \neq e$ for which $z<C$.

THEOREM 2. Let $(X, T, I)$ be a transformation group, where $X$ has an order $\leqq$ which satisfies (b)-(d) and $\left(\mathrm{e}_{\mathrm{s}}\right)$, and $T$ acts as a compact group of order isomorphisms on $X$. Let $x \in X$ with $x \neq e$. Let

$$
M(T x)=\{y ; y \leqq T x\}=\cap\{M(y) ; y \in T x\} .
$$

Then $T$ leaves each point of $M(T x)$ fixed. Furthermore $M(T x)$ is an infinite set.

Proof. The set $M(T x)$ is a $T$-invariant chain by axiom (d). Let $z \in M(T x)$. Then $T z$ is a compact subchain of $A$, and since (f) holds for $\leqq$ without assuming (a), $T z$ contains a maximal element $m$. Since $T z$ is a chain, $m$ is the largest element of $T z$, hence is fixed under $T$. Thus the orbit of $z$ contains a fixed point under $T$, so that $T$ leaves $z$ fixed. Now $\left(\mathrm{g}_{\mathrm{s}}\right)$ also holds for $\leqq$, so that the set $M(T x)$ is infinite, and the proof is complete.

In what follows, let $X$ be a nontrivial Hausdorff continuum. If $e \in X$, then $e$ is an end point of $X$ if, given an open set $U$ with $e \in U$, there exists $y \in U$ such that $y \neq e$ and

$$
X-y=V \cup W, e \in V \subset U,(\bar{V} \cap W) \cup(V \cap \bar{W})=\varnothing .
$$

If $x \in X$, let $E(e, x)=\{e, x\} \cup\{z ; z$ separates $e$ and $x$ in $X\}$ Given two points $x, y \in X$, define $x \leqq y$ if and only if $x \in E(e, y)$. Then $\leqq$ satisfies (b)-(e) and $\left(\mathrm{e}_{\mathrm{s}}\right)$. Furthermore, a homeomorphism: $X \rightarrow X$ which leaves $e$ fixed is an order isomorphism. If in addition $X$ is locally connected, $\leqq$ satisfies (a), and the results of this section apply to such a space. Hence if $(X, T, \Pi)$ is a transformation group, where $X$ is locally connected and $T e=e$, and if there is a closed nonempty $T$-invariant subset $A \subset X$ such that $e \notin A$, then $T$ has a fixed point other than $e$. 
From Theorem 2 we obtain

Corollary 2.1. Let $(X, T, \Pi)$ be a transformation group, where $X$ is a nontrivial Hausdorff continuum and $T$ is a compact group which leaves an end point $e$ of $X$ fixed. If $x \in X$ and $x \neq e$, let

$$
E(e, T x)=\{y ; y \text { separates } e \text { and } T x \text { in } X\} .
$$

Then $T$ leaves each point of $E(e, T x)$ fixed.

We will call a metric continuum a dendrite if each two distinct points of the continuum is separated by a third point of the continuum. It is known [10] that each point of a dendrite is either a cut point or an end point.

Corollary 2.2. Let $X$ be a dendrite with a finite number, $N$, of end points. Then the only compact groups which can act effectively on $X$ are the subgroups of $S_{N}$, the permutation group on $N$ symbols.

Proof. Let $E$ be the set of end points of $X$ and $T$ be a compact group which acts effectively on $X$. Then for each $t \in T$, the restriction, $t \mid E$, of $t$ to $E$ is in $S_{N}$, and the mapping $t \rightarrow t \mid E$ is a homomorphic mapping of $T$ onto a subgroup of $S_{N}$.

Let $P$ be the set of all elements of $T$ which leave each point of $E$ fixed. $P$ is a closed subgroup of $T$, and since $X=\cup\{E(x, y) ; x, y \in E\}$, it follows from Corollary 2.1 that $P$ leaves each element of $X$ fixed, and because $T$ is effective, $P$ is the identity alone. Thus if $t \mid E=$ $s \mid \mathbb{E}$, then $s^{-1} t \in P$, hence $s=t$. Thus the mapping $t \rightarrow t \mid E$, all $t \in T$, is an isomorphism.

3. In this section, $X$ will denote a nontrivial locally connected Hausdorff continuum, and $T$ is a group which leaves an end point $e$ of $X$ fixed. We remark that all the results of this section hold when $X$ is arcwise connected but not necessarily locally connected (we replace the remark immediately preceding Corollary 2.1 by Wang's Lemma, [9]).

Lemma 3.1. Let $(X, T, \Pi)$ be a transformation group, where $T$ is connected. Then $T$ has a fixed point other than $e$.

Proof. Since $X$ contains at least two noncut points, [8], let $x \neq e$ be another noncut point, and

$$
X-z=U \cup V, e \in U, x \in V,(\bar{U} \cap V) \cap(U \cup \bar{V})=\varnothing ;
$$

now $T x$ contains only noncut points, and so $z \notin T x$ since $z$ is a cut point. Since $T x$ is connected, it follows that $T x \subset V$. Because 
$V \cup\{z\}$ is closed, we have $\overline{T x} \subset V \cup\{z\}$. We have found a nonempty closed $T$-invariant set not containing $e$, so that the remark preceding Corollary 2.1 applies.

THeOREM 3. Let $(X, T, \Pi)$ satisfy the hypothesis of Lemma 3.1. Either $e$ is the only noncut point in one of its neighborhoods, or else $T$ has infinitely many fixed points.

Proof. We use the order and notation of $\S 2$. Let $x_{0}$ be a noncut point of $X$ with $x_{0} \neq e$. From the proof of Lemma 2.1, we see that $\mathscr{M}\left(\overline{T x}_{0}\right)$ is a fixed point different from $e$. Let $A_{1}=\mathscr{C}\left(\overline{T x}_{0}\right) \cup{\overline{T x_{0}}}_{0}$. Since $e$ does not belong to the closed set $A_{1}$, we may find $z \in X$ for which

$$
X-z=U \cup V, e \in U, A_{1} \subset V,(\bar{U} \cap V) \cup(U \cap \bar{V})=\varnothing .
$$

Suppose every neighborhood of $e$ contains a cut point other than $e$, and let $x_{1} \in U$ be such a point. Since $z$ is a cut point, $z \notin T x_{1}$ so that ${\overline{T x_{1}}}_{1} \subset U \cup\{z\}$. Furthermore, a separation argument shows that if $x \in \overline{T x_{1}}$, then $M(x) \subset U \cup\{z\}$ so that $\mathscr{C l}\left(\overline{T x_{1}}\right) \subset U \cup\{z\}$. Since $\mathscr{C l}\left(T x_{0}\right) \in V$, we have $\mathscr{C}\left(T x_{1}\right) \neq \mathscr{C l}\left(T x_{0}\right)$. Set

$$
A_{2}=\overline{T x_{0}} \cup \overline{T x_{1}} \cup \mathscr{C l}\left(\overline{T x_{0}}\right) \cup \mathscr{C l}\left(\overline{T x_{1}}\right),
$$

and complete the proof by induction.

THEOREM 4. Let $(X, T, \Pi)$ be a transformation group, with $T \approx A H$, where $A$ is an abelian group which is generated by a compact subset and lies in the center of $T$, and $H$ is a connected subgroup. Then $T$ has a fixed point other than $e$.

Proof. Let $X$ be a fixed point under $A$, where $x \neq e$. Then $\overline{T x}=\overline{H x}$ is connected. If $e \notin \overline{H x}$, we are finished (in view of previous results). If $e \in \overline{H x}$, since $\overline{H x}$ is a nontrivial Hausdorff continuum, $\bar{H} \bar{x}$ contains a noncut point $y \neq e$. Then for some $z \in X$,

$$
X-z=U \cup V, e \in U, y \in V,(\bar{U} \cap V) \cup(U \cap \bar{V})=\varnothing .
$$

Because $\overline{H x}$ is connected, $z$ is a cut point of $H x$. Since $H y$ contains only noncut points of $\overline{H x}, z \notin H y$, and $\overline{H y} \subset(V \cap \overline{H x}) \cup\{z\}$, for the last set is closed in $X$. Now $A$ lies in the center of $T$, hence every point of $H x$ is fixed under $A$, so that $\overline{H y}$ is a $T$-invariant set not containing $e$. By the remark at the end of $\S 2$, the proof is complete.

The author is indebted to Professor Hsin Chu for his encouragement during the preparation of this paper, and to the referee for pointing out two previously overlooked generalizations in the theorems. 


\section{REFERENCES}

1. Hsin Chu, Fixed points in a transformation group (to appear in the Pacific J. Math.)

2. A note on transformation groups with a fixed end point (to appear in the Proc. Amer. Math. Soc.)

3. W. H. Gottschalk and G. A. Hedlund, Topological Dynamics, Amer. Math. Soc. Colloq. Publ., XXXVI, Providence, 1955.

4. William J. Gray, Topological transformation groups with a fixed end point (pending with the Proc. Amer. Math. Soc.)

5. J. G. Hocking and G. S. Young, Topology, Addison-Wesley, Reading, 1961.

6. A. D. Wallace, $A$ fixed point theorem, Bull. Amer. Math. Soc. 51 (1945), 413-416.

7. - Group invariant continua, Fund. Math. 36 (1949), 119-124.

8. Monotone transformations, Duke Math. J. 9 (1942), 487-506.

9. H. C. Wang, A remark on transformation groups leaving fixed an end point, Proc. Amer. Math. Soc. 3 (1952), 548-549.

10. R. L. Wilder, Topology of Manifolds, Amer. Math. Soc. Colloq. Publ., XXXII, Providence, 1949.

Received October 24, 1965, and in revised form February 5, 1966.

UNIVERSITY OF ALABAMA 



\section{PACIFIC JOURNAL OF MATHEMATICS}

\section{EDITORS}

H. SAMELSON

Stanford University

Stanford, California

J. P. JANS

University of Washington

Seattle, Washington 98105

\section{J. DuGUNDJI}

University of Southern California

Los Angeles, California 90007

RICHARD ARENS

University of California

Los Angeles, California 90024

\section{ASSOCIATE EDITORS}

E. F. BECKENBACH
B. H. NEUMANN

F. WOLF

K. YoSIDA

\section{SUPPORTING INSTITUTIONS}

\author{
UNIVERSITY OF BRITISH COLUMBIA \\ CALIFORNIA INSTITUTE OF TECHNOLOGY \\ UNIVERSITY OF CALIFORNIA \\ MONTANA STATE UNIVERSITY \\ UNIVERSITY OF NEVADA \\ NEW MEXICO STATE UNIVERSITY \\ OREGON STATE UNIVERSITY \\ UNIVERSITY OF OREGON \\ OSAKA UNIVERSITY \\ UNIVERSITY OF SOUTHERN CALIFORNIA
}

\author{
STANFORD UNIVERSITY \\ UNIVERSITY OF TOKYO \\ UNIVERSITY OF UTAH \\ WASHINGTON STATE UNIVERSITY \\ UNIVERSITY OF WASHINGTON \\ AMERICAN MATHEMATICAL SOCIETY \\ CHEVRON RESEARCH CORPORATION \\ TRW SYSTEMS \\ NAVAL ORDNANCE TEST STATION
}

Mathematical papers intended for publication in the Pacific Journal of Mathematics should be typewritten (double spaced). The first paragraph or two must be capable of being used separately as a synopsis of the entire paper. It should not contain references to the bibliography. Manuscripts may be sent to any one of the four editors. All other communications to the editors should be addressed to the managing editor, Richard Arens at the University of California, Los Angeles, California 90024 .

50 reprints per author of each article are furnished free of charge; additional copies may be obtained at cost in multiples of 50 .

The Pacific Journal of Mathematics is published monthly. Effective with Volume 16 the price per volume (3 numbers) is $\$ 8.00$; single issues, $\$ 3.00$. Special price for current issues to individual faculty members of supporting institutions and to individual members of the American Mathematical Society: $\$ 4.00$ per volume; single issues $\$ 1.50$. Back numbers are available.

Subscriptions, orders for back numbers, and changes of address should be sent to Pacific Journal of Mathematics, 103 Highland Boulevard, Berkeley 8, California.

Printed at Kokusai Bunken Insatsusha (International Academic Printing Co., Ltd.), No. 6, 2-chome, Fujimi-cho, Chiyoda-ku, Tokyo, Japan.

\section{PUBLISHED BY PACIFIC JOURNAL OF MATHEMATICS, A NON-PROFIT CORPORATION}

The Supporting Institutions listed above contribute to the cost of publication of this Journal, but they are not owners or publishers and have no responsibility for its content or policies. 


\section{Pacific Journal of Mathematics

Vol. 19, No. $3 \quad$ July, 1966

S. J. Bernau, The spectral theorem for unbounded normal operators .......

Lu-san Chen, Asymptotic behavior of solutions of parabolic equations of

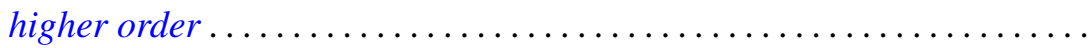

Lawrence William Conlon, An application of the Bott suspension map to the

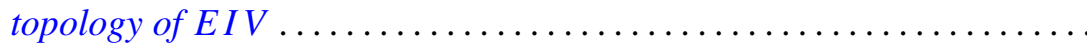

Neal Eugene Foland and John M. Marr, Sets with zero-dimensional kernels .........................................

Stanley Phillip Franklin and R. H. Sorgenfrey, Closed and image-closed

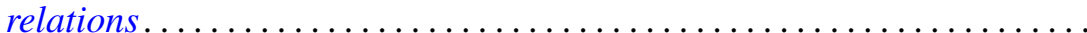

William Jesse Gray, A note on topological transformation groups with a

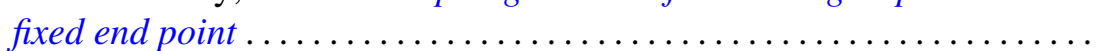

Myron Goldstein, $K$ - and L-kernels on an arbitrary Riemann surface ...... 449

George Joseph Kertz and Francis Regan, The exponential analogue of a generalized Weierstrass series .............................

Walter Leighton, On Liapunov functions with a single critical point ........ 467

Bernard Werner Levinger and Richard Steven Varga, On a problem of $O$.

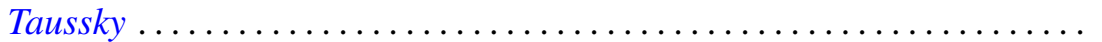

Lowell Duane Loveland, Tame subsets of spheres in $E^{3} \ldots \ldots \ldots \ldots \ldots . .489$

Erik Andrew Schreiner, Modular pairs in orthomodular lattices ......... 519

K. N. Srivastava, On dual series relations involving Laguerre polynomials ...............................

Arthur Steger, Diagonability of idempotent matrices.....

Walter Strauss, On continuity of functions with values in various Banach spaces...

Robert Vermes, On the zeros of a linear combination of polynomials ...

Elliot Carl Weinberg, On the scarcity of lattice-ordered matrix rings ....

Harold Widom, Toeplitz operators on $H_{p} \ldots \ldots \ldots \ldots \ldots$

Neal Zierler, On the lattice of closed subspaces of Hilbert space...

Irving Leonard Glicksberg, Correction to: "Maximal algebras and a theorem of Rado"

John Spurgeon Bradley, Correction to: "Adjoint quasi-differential operators of Euler type"

William Branham Jones, Erratum: "Duality and types of completeness in locally covex spaces".

Stanley P. Gudder, Erratum: "Uniqueness and existence properties of bounded observables" 